\title{
Can we extend thrombolysis indications for acute ischaemic stroke? The IST-3 study
}

\author{
Marta Del Medico • Antonella Bonura • \\ Gruppo di Autoformazione Metodologica (GrAM)
}

Received: 11 December 2012/ Accepted: 22 December 2012/Published online: 1 March 2013

(C) SIMI 2013

\section{Background}

Each year, about 22 million people have a stroke worldwide, of whom 4 million reside in high-income countries. The burden of ischaemic stroke among the elderly is large and increasing; it is estimated that annually ischaemic stroke affects about a million people older than 80 years in high-income countries and about 3 million in low-income and middle-income countries [1].

So far intravenous thrombolysis is indicated for patients with ischaemic stroke, aged less than 80 , within $4.5 \mathrm{~h}$ from the occurence of neurologic signs, without any contraindications [2].

Since many patients with acute ischaemic stroke, according to current guidelines, cannot be candidated for thrombolysis, it would be appealing to extend its indications.

Although thrombolysis has been proved to ameliorate morbidity in acute ischaemic stroke, it increases cerebral haemorrhage; so, before extending thrombolysis indications, strong evidence of benefits is needed [3].

\section{Summary}

The third international stroke trial (IST-3) is a multicentre, pragmatic, open-treatment study which randomised 3,035

\section{Del Medico $(\square)$}

U.O Medicina 2, Ospedale L. Sacco,

University of Milan, Milan, Italy

e-mail: madelme@gmail.com

A. Bonura

U.O Medicina 3, Ospedale L. Sacco,

University of Milan, Milan, Italy patients to $0.9 \mathrm{mg} / \mathrm{kg}$ intravenous recombinant tissue plasminogen activator (rt-PA) or to control [1].

The primary endpoint was the proportion of patients alive and independent at 6 months, as defined by an Oxford Handicap Score (OHS) of 0-2. The main secondary endpoints were mortality and morbidity at 7 days. Initially, there was a pilot phase, double blinded and placebo controlled $(<10 \% \mathrm{pts})$, and then authors shifted to open treatment. The eligibility criteria can be summarized in terms of the uncertainty principle. Briefly, patients were eligible according to the following criteria: they had symptoms and signs of clinically definite acute stroke; the time of stroke onset was known; treatment could be started within $6 \mathrm{~h}$ of onset; and CT or MRI had reliably excluded both intracranial haemorrhage and structural brain lesions, which could mimic stroke. In addition, if the patient had a clear indication for intravenous thrombolysis with rt-PA, they were to be treated in accordance with local guidelines. Equally, if the patient had a clear contraindication to treatment, they were not to be entered in the trial. The data were analysed following the intention-to-treat principles.

Of notice that among 3,035 patients enrolled, $95 \%$ did not meet local European guidelines for thrombolysis, $72 \%$ were treated more than $3 \mathrm{~h}$ after stroke onset, $53 \%$ aged more than 80 and $5 \%$ had a NIHSS greater than 25 .

At 6 months, 554 (37\%) patients in the rt-PA group versus $534(35 \%)$ in the control group were alive and independent [OHS 0-2; adjusted odds ratio (OR) 1.13, $95 \%$ CI $0.95-1.35, p=0.181$ ). Fatal or non-fatal symptomatic intracranial haemorrhage within 7 days occurred in $104(7 \%)$ patients in the rt-PA group versus $16(1 \%)$ in the control group (adjusted OR 6.94, $95 \%$ CI 4.07-11.8, $p<0.001)$. More deaths occurred within 7 days in the rtPA group [163 (11\%)] than in the control group [107 (7 \%), adjusted OR 1.60, $95 \%$ CI 1.22-2.08, $p=0.001]$, 
but between 7 days and 6 months, there were fewer deaths in the rt-PA group than in the control group, so that by 6 months, similar numbers, in total, had died [408 (27\%) in the rt-PA group vs. 407 (27\%) in the control group]. Finally, an ordinal logistic analysis showed a significant shift in OHS scores (common OR 1.27, $95 \%$ CI 1.10-1.47, $p=0.001)$.

Authors concluded that thrombolysis within $6 \mathrm{~h}$ improved functional outcome and that this benefit did not seem to be diminished in elderly patients.

\section{Strengths of the study}

- It deals with a clinically relevant problem. Stroke is a common disease, increasing with age, a leading cause of death and disability all over the world.

- Large number of enrolled patients

- The pragmatic feature of this trial gives a greater external validity to its results

- The trial had a long-term follow-up and only a few patients were lost

\section{Weaknesses of the study}

- Although the primary end-point was not met, the authors based their conclusions on subgroup and secondary analysis

- In many patients, the follow-up for disability was conducted by phone calls

- The sample size was re-estimated during the trial

\section{Question marks}

- It would be interesting to have some more data about patients comorbidities, in order to better compare the study population to patients seen in the everyday clinical practice

- We wonder if switching from the double-blind pilot to the open treatment phase could affect some study results
- It could be interesting to know the rehabilitation programs followed by patients in the two groups. Since the favourable impact of rehabilitation on disability, we wonder if any difference between groups could affect the primary endpoint

- Since the trial has been conducted for 12 years, we wonder if in such a long period physicians could change their management of acute stroke patients

\section{Clinical bottom line}

So far there is not enough evidence to say that thrombolysis within $6 \mathrm{~h}$ in patients older than 80 improves functional outcome and survival. On the contrary, there is evidence that symptomatic intracranial haemorrhage occurred more frequently in patients treated with rt-PA, and that mortality in the short-term period is higher in this group of patients.

\section{Sponsorship}

Data collection and analysis were not performed by any sponsor. However, some sponsors have paid travel costs, conferences and lectures fees to many authors, or provided grants and donations for scanner purchase.

Conflict of interest None.

\section{References}

1. IST-3 collaborative group (2012) The benefits and harms of intravenous thrombolysis with recombinant tissue plasminogen activator within $6 \mathrm{~h}$ of acute ischaemic stroke (the third international stroke trial [IST-3]): a randomised controlled trial. Lancet 379(9834):2352-2363. doi:10.1016/S0140-6736(12)60768-5

2. Guidelines for the Early Management of Adults With Ischemic Stroke (2007) AHA/ASA guidelines. Stroke 38:1655-1711

3. Wardlaw JM, Murray V, Berge E, Del Zoppo GJ (2009) Thrombolysis for acute ischemic stroke. Cochrane Database Syst Rev 4:CD000213 